\title{
On action-angle coordinates and on the Poincaré coordinates
}

\author{
Jacques Féjoz \\ Université Paris-Dauphine, Ceremade \\ and Observatoire de Paris, IMCCE \\ jacques.fejoz@dauphine.fr
}

January 2, 2014

To Alain Chenciner's birthday, with admiration and gratitude

Keywords: Hamiltonian system, Lagrangian fibration, action-angle coordinates, Liouville-Arnold theorem, adiabatic invariants, Kepler problem, two-body problem, Poincaré coordinates, planetary problem, first integral, integrability, perturbation theory

MSC 2010: 01-01, 37-03, 37C80, 37J35, 70-03, 70H06, 70H15

Abstract: This article is a review of two related classical topics of Hamiltonian systems and celestial mechanics. The first item deals with the existence and construction of action-angle coordinates, which we describe emphasizing the role of the natural adiabatic invariants " $\oint_{\gamma} p d q$ ". The second item is the construction and properties of the Poincaré coordinates in the Kepler problem, adapting the principles of the former item, in an attempt to use known first integrals more directly than Poincaré did.

\section{Action-angle coordinates}

\subsection{Main statement and comments}

Let $(M, \omega)$ be a symplectic manifold of dimension $2 n$ and $F: M \rightarrow B$ be a fibration whose fibers $M_{b}, b \in B$, are Lagrangian 11 compact and connected submanifolds of $M$. Roughly speaking, the theorem of the action-angle coordinates says that locally in the neighborhood of a base point, the universal model for $F$ is the canonical projection

$$
F^{o}: M^{o}=B^{o} \times \mathbb{T}^{n} \rightarrow B^{o}, \quad(I, \theta) \mapsto I,
$$

\footnotetext{
${ }^{1}$ Sometimes, such a fibration is called a (reducible) real polarization of $M[49$.
} 
where $B^{o}$ is a domain of $\mathbb{R}^{n}, \mathbb{T}^{n}=\mathbb{R}^{n} / \mathbb{Z}^{n}$ is the $n$-torus and $M^{o}$ is endowed with the standard symplectic form $\omega^{o}=\sum_{j} d I_{j} \wedge d \theta_{j}$. All maps considered here are smooth.

Theorem 1. For every $b \in B$, there exist a neighborhood $B(b)$ of $b$ in $B$, a domain $B^{o}$ of $\mathbb{R}^{n}$, a diffeomorphism $I: B(b) \rightarrow B^{o}$ and a symplectic diffeomorphism $(I, \theta):\left.M\right|_{B(b)} \rightarrow M^{o}$ above $I$, i.e. such that the following diagram commutes:

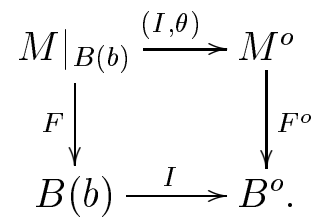

The coordinates $I$ and $\theta$ are respectively called action and angle coordinates.

Example 1. Let $F=\left(F_{1}, \ldots, F_{n}\right): M \rightarrow \mathbb{R}^{n}$ be a family of $n$ independent Hamiltonians in involution:

$$
\begin{cases}\left\{F_{j}, F_{j}\right\}=0 & (\forall i, j) \\ \operatorname{rk} F^{\prime}(x)=n & (\forall x \in M),\end{cases}
$$

such that the levels of $F$ are compact and connected. Any of the components $F_{i}$ 's is then often called an integrable Hamiltonian system.2 Due to a classical theorem of Ehresman [17, 32, $F$ is a fibration. The tangent space of its fibers is generated by the Hamiltonian vector fields of $F_{i}, i=1, \ldots, n$. Since the $F_{i}$ commute, fibers are Lagrangian and the hypotheses of the theorem are satisfied.

The history of action-angle coordinates has known several stages, which can be sketched as follows.

- Early versions of Liouville [36] or Jacobi [25] focus on the possibility of local integration of a Hamiltonian system (or, more generally, of an ordinary differential equation) by quadrature. They single out the hypothesis of $n$ commuting independent first integrals, but they do not provide a a topological description of the quasiperiodic tori foliation of the phase space.

- In the course of the 19th century, astronomers fill this gap, realizing the importance and the non-genericity of the foliation of the phase space in "multiply periodic" solutions 3 in particular in integrable approximations of the planetary problem [43].

\footnotetext{
${ }^{2}$ In addition to the integrability of differential equations by quadrature, integrability may also refer to Pfaff systems satisfying the hypotheses of the Frobenius theorem or, more generally, to geometric structures satisfying some flatness condition [45. Also, some authors speak of completely integrable Hamiltonian systems, while they keep the phrase (non completely) integrable for Hamiltonian systems with any intermediate number $k \in\{1, \ldots, n\}$ of first integrals, thus being not integrable...

${ }^{3}$ Today, such solutions are generally called 'quasiperiodic. Yet some authors rather call them conditionally periodic, keeping the adjective "quasiperiodic" for conditionally periodic with an irrational frequency vector [7].
} 
- Several versions of the theorem of the action-angle coordinates, in the early 20th century, are related to adiabatic invariants and the Bohr-Sommerfeld quantization (see remark 3). They are due to Gibbs-Hertz [47, Bd 1, p. 535] (adiabatic invariance of the volume), Burgers [11] (adiabatic invariance of the $\oint p_{i} d q_{i}$ for decoupled systems) and others. Poincaré [30] suggests to replace the Bohr-Sommerfeld rule of quantization by a rule which is invariant, substituting an integral invariant for the $p_{i} d q_{i}$ 's; he also raises the issue of the question of the uniqueness of the system of adiabatic invariants (see also Einstein's quantization [18]). Epstein discusses how degeneracy induces ambiguity in the choice of adiabatic invariants and thus in quantization [19]. Levi-Civita [34] and Mineur [39] seemingly prove the modern statement on action-angle coordinates.

- Landau-Lipschitz treats of adiabatic invariants in a way close to Mineur [29]. Arnold uses a more modern geometric language [3, 6].

- Some unnecessary hypotheses, such as the functional independence of the action variables and the exactness of the symplectic form, are removed in [16, 26, 37], with variants [7, 24, 35]. Usually these proofs build some angle coordinates by straightening the period lattice of the flow of the first integrals, and then define the action variables as the variables which are symplectically conjugate to the angles. In order to prove integrability by quadrature, one eventually needs to show how these coordinates relate to the natural adiabatic invariants (e.g., see [16]).

- Generalizations in several directions: refined integrability properties [4, 27, geometric quantization [49], globalness and monodromy of the action [15, [16], singular fibrations [33], or, non-commuting integrals (the question then interestingly relates to weak KAM theory) [12].

Here we will review the proof of theorem 1 in the lines of Duistermaat and Guillemin-Sternberg [16, 24], with only minor differences aiming at practical computations, in relation to the second section of this article.

\subsection{Toral actions}

Lemma 1. Let $V$ be a real vector space of finite dimension n, acting on a compact connected manifold $X$ of dimension $n$. The action is transitive if and only if it is infinitesimally transitive, in which case $X$ is diffeomorphic to $\mathbb{T}^{n}$.

For all $x \in X$, let $\rho_{x}: V \rightarrow X, v \mapsto \rho_{x}(v)=v \cdot x$. Recall that the action is transitive if the orbit $\rho_{x}(V)$ is the entire $X$ for all $x \in X$, and that it is infinitesimally transitive if $\rho_{x}^{\prime}(0): V \rightarrow T_{x} X$ (derivative of $\rho_{x}$ at $v=0$ ) is an isomorphism for all $x \in X$. 
Proof. By the definition of an action, if $x \in X$ and $v \in V$ we have $\rho_{x}(v+h)=$ $v \cdot \rho_{x}(h)$ for all $h \in V$. Hence, by differentiating with respect to $h$,

$$
\rho_{x}^{\prime}(v)=v^{\prime}(x) \cdot \rho_{x}^{\prime}(0)
$$

and, since $v^{\prime}(x)$ is an isomorphism, $\rho_{x}^{\prime}(0)$ is an isomorphism if and only if, for all $v, \rho_{x}^{\prime}(v)$ is one.

So, if the action is infinitesimally transitive, by the inverse function theorem, the orbit $\rho_{x}(V)$ of $x$ is open. Since $X$ is compact, $X$ is covered by a finite union of orbits. Since $X$ is connected, there can only be one orbit. Thus the action is transitive.

Conversly, if the action is not infinitesimally transitive, due to the remark above, $\rho_{x}^{\prime}(v)$ is invertible for no $v \in V$, so the whole orbit $\rho_{x}(V)$ consists of critical values of $\rho_{x}$ and, by Sard's theorem, has measure zero. So, the action is not transitive.

Assume again that the action is transitive. Let $x$ and $y$ be any two points of $X$. Since the action is transitive, the stabilizers of $x$ and $y$ are conjugate. Since $V$ is an Abelian group, the stabilizers agree. Let $L \subset V$ be the common stabilizer of points of $X$. As already mentioned, due to the infinitesimal transitivity of the action, $\rho_{x}$ is a local diffeomorphism in the neighborhood of $v \mapsto v \cdot x$ for every $x$ and $v$. Hence $L$ has only isolated points, hence $L$ is discrete. So, $L$ is a lattice [8, 46]. Since $X$ is compact, $L$ is a maximal lattice. So, $V / L$ is compact, hence an $n$-torus. Since $X$ is diffeomorphic to $V / L, X$ itself is an $n$-torus.

The above stabilizer $L$ is called the period lattice of the action.

Example 2. Let $\xi_{1}, \ldots, \xi_{n}$ be $n$ vector fields on $X$, commuting and everywhere independent:

$$
\left\{\begin{array}{l}
{\left[\xi_{i}, \xi_{j}\right]=0 \quad(\forall i, j)} \\
\operatorname{Span}\left(\xi_{1}(x), \ldots, \xi_{n}(x)\right)=T_{x} X \quad(\forall x \in X) .
\end{array}\right.
$$

Then the "joint flow of the $\xi_{i}$ 's"

$$
\mathbb{R}^{n} \times X \rightarrow X, \quad(v, x) \mapsto \phi_{v}^{\xi}(x)=\varphi_{v_{1}}^{\xi_{1}} \circ \cdots \circ \varphi_{v_{n}}^{\xi_{n}}(x),
$$

where $\varphi_{v_{i}}^{\xi_{i}}$ is the time- $v_{i}$ of the flow of $\xi_{i}$, is an infinitesimally transitive action.

\subsection{Lagrangian fibrations}

We now aim at proving theorem 1, Let $F: M \rightarrow B$ be as in the statement.

Lemma 2 ([24]). There is a transitive action of $T^{*} B$ on $M$ and fibers of $F$ are $n$-tori.

Proof. Let $b \in B$ and $x \in M_{b}$. The cotangent map $F^{*}(x): T_{b}^{*} B \rightarrow T_{x}^{*} M$ composed with the isomorphism $T_{x}^{*} M \rightarrow T_{x} M$ induced on the left by $\omega$ (inverse of $X_{x} \mapsto$ 
$\left.i\left(X_{x}\right) \omega\right)$ allows us to associate to a cotangent vector $\beta_{b} \in T_{b}^{*} B$ a vector $\hat{\beta}_{x}$ tangent to $M$ at every point $x$ in the fiber of $b$, characterized by the equation

$$
i\left(\hat{\beta}_{x}\right) \omega=F^{*}(x) \cdot \beta_{b} .
$$

Since the 1-form $F^{*}(x) \cdot \beta_{b}$ vanishes on vertical vectors, it induces an element of the normal bundle $N_{x}^{*}\left(M_{b}\right)=T_{x}^{*} M / \operatorname{Ker} T_{x} F$ of the fiber at $x$. Since the fiber is Lagrangian (otherwise said, equal to its own $\omega$-orthgogonal), $\omega$ identifies $N_{x}^{*}\left(M_{b}\right)$ with the vertical tangent space $T_{x}\left(M_{b}\right)$. So, $\hat{\beta}_{x}$ is vertical and the map

$$
T_{b}^{*} B \rightarrow T_{x}\left(M_{b}\right), \quad \beta_{b} \mapsto \hat{\beta}_{x}
$$

is an isomorphism. Since the fibers are compact, the vector field $\hat{\beta}$ can be exponentiated into a fibered diffeomorphism, which we will simply denote by $\beta$.

For this construction to define an action, we need that, if $\beta_{1}, \beta_{2} \in T_{b}^{*} B$, their associated diffeomorphisms commute. Let $h_{1}$ and $h_{2}$ be functions on $B$ such that $\beta_{i}=h_{i}^{\prime}(b), i=1,2$. If $x \in M_{b}$,

$$
\left(h_{i} \circ F\right)^{\prime}(x)=F^{*}(x) \cdot \beta_{i},
$$

hence, the vector field

$$
\hat{\beta}_{i}(x)=X_{F^{*} h_{i}}(x)
$$

is the restriction of a Hamiltonian vector field and, since fibers are Lagrangian,

$$
\left[\hat{\beta}_{1}, \hat{\beta}_{2}\right]_{x}=\left\{F^{*} h_{1}, F^{*} h_{2}\right\}_{x}=\omega_{x}\left(X_{F^{*} h_{1}}, X_{F^{*} h_{2}}\right)=0 .
$$

We have thus defined an action of $T_{b} B$ on the fiber $M_{b}$, for all $b \in B$. The action is infinitesimally transitive. Hence, according to lemma 1, the action is transitive and fibers of $F$ are $n$-tori. .

A first consequence of lemma 2 (and its proof) is the existence of local Lagrangian submanifolds of $M$ which are transverse to the fibers.

Lemma 3. If $\beta$ is a 1-form on $B$,

$$
\beta^{*} \omega=\omega+F^{*} d \beta
$$

where on the left hand-side $\beta$ is thought of as a diffeomorphism of $M$. Consequently, there exists a section of $F$ whose image is Lagrangian.

Proof. If $\beta$ is a 1 -form on $B$,

$$
\begin{array}{rlr}
\beta^{*} \omega & =\omega+\int_{0}^{1}(t \beta)^{*} L_{\hat{\beta}} \omega d t & \\
& =\omega+\int_{0}^{1}(t \beta)^{*} d i(\hat{\beta}) \omega d t & \text { (Cartan formula) } \\
& =\omega+\int_{0}^{1}(t \beta)^{*} F^{*} d \beta d t & \text { (by definition of } \hat{\beta} \text { ) } \\
& =\omega+F^{*} d \beta & \text { (because } F \circ(t \beta)=F) .
\end{array}
$$


Now, let $\sigma_{0}: B \rightarrow M$ be a section of $F$. There is a 1 -form $\beta$ on $B$ such that $\sigma_{0}^{*} \omega=d \beta$. From lemma 2, and using the fact that $F \circ \sigma_{0}=\mathrm{id}$,

$$
\left((-\beta) \circ \sigma_{0}\right)^{*} \omega=\sigma_{0}^{*}\left(\omega-F^{*} d \beta\right)=0 .
$$

So, the section $\sigma=(-\beta) \cdot \sigma_{0}$ is Lagrangian.

The next lemma is a key step towards understanding the structure of $M$. We endow $T^{*} B$ with its canonical symplectic form, which we denote by $\omega^{o}$.

Remark 1. In the construction of lemma 2, we may replace $F$ by the canonical projection $\pi: T^{*} B \rightarrow B$. If $\beta \in T_{b}^{*} B$, the vertical vector field $\hat{\beta}^{o}$ along the fiber of $b$ is defined by $i\left(\hat{\beta}^{o}\right) \omega^{o}=\pi^{*} \cdot \beta$. If one identifies the tangent space at $\gamma \in T_{b}^{*} B$ of $T_{b}^{*} B$ with $T_{b}^{*} B, \hat{\beta}^{o}$ identifies with $\beta$, seen as a constant vertical vector field. Thus $\hat{\beta}^{o}$ exponentiates (despite the fiber being not compact) into a diffeomorphism $\beta^{o}$ of $T_{b}^{*} B$, which is just the vertical translation:

$$
\beta^{o} \cdot \gamma=\beta+\gamma
$$

Lemma 4. Let $\sigma$ be a Lagrangian section of $F$. The map

$$
\chi: T^{*} B \rightarrow M, \quad \beta_{b} \mapsto \beta_{b} \cdot \sigma(b)
$$

is symplectic.

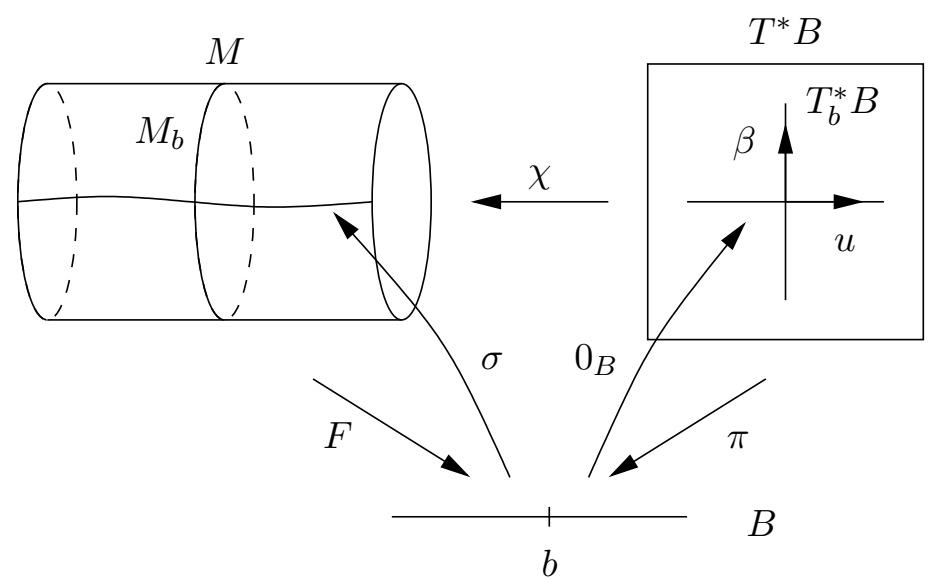

Proof. Let us first prove that $\chi$ is symplectic at some point $(b, 0)$ of the zero-section $0_{B}$ of $T^{*} B$. The tangent space of $T^{*} B$ at $(b, 0)$ splits into its horizontal and vertical subspaces, $T_{(b, 0)} 0_{B}$ and $T_{(b, 0)} T_{b}^{*} B$, both Lagrangian. The derivative $\chi^{\prime}$ maps these subspaces respectively to the tangent spaces at $\sigma(b)$ of the image of $\sigma$ and of the fiber $M_{b}$, both of which are Lagrangian too. So it is enough to check that

$$
\chi^{*} \omega(\beta, u)=\omega^{o}(\beta, u)
$$

with $\beta \in T_{(b, 0)} T_{b}^{*} B=T_{b}^{*} B$ and $u \in T_{(b, 0)} 0_{B}=T_{b} B$. For such vectors $\beta$ and $u$, on the one hand we have 4

$$
\omega^{o}(\beta, u)=\beta \cdot u \text {. }
$$

\footnotetext{
${ }^{4}$ Here we choose between the two possible signs of the canonical symplectic form.
} 
On the other hand, we have

$$
\chi^{\prime} \cdot \beta=\hat{\beta}_{\sigma(b)} \quad \text { and } \quad \chi^{\prime} \cdot u=\sigma^{\prime} \cdot u,
$$

hence

$$
\begin{aligned}
\chi^{*} \omega(\beta, u) & =\omega\left(\hat{\beta}_{\sigma(b)}, \sigma^{\prime} \cdot u\right) & \\
& =F^{*} \beta \cdot\left(\sigma^{\prime} \cdot u\right) & \text { (by definition of } \hat{\beta} \text { ) } \\
& =\beta \cdot u & \text { (using that } F \circ \sigma=\mathrm{id}) .
\end{aligned}
$$

Let us now consider any point $\gamma \in T_{b}^{*} B$ not necessarily on the zero-section. First notice that

$$
\begin{aligned}
\beta \circ \chi(\gamma) & =\beta \cdot(\gamma \cdot \sigma(b)) \\
& =(\beta+\gamma) \cdot \sigma(b) \\
& =\chi \circ \beta^{o}(\gamma) .
\end{aligned}
$$

Now, if $u$ and $v$ are two vectors tangent to $T^{*} B$ at $\gamma$, they are of the form

$$
u=\gamma^{o \prime} \cdot u_{1} \text { and } \quad v=\gamma^{o \prime} \cdot v_{1}
$$

with $u_{1}, v_{1}$ tangent to $T^{*} B$ at $(b, 0)$, and

$$
\begin{aligned}
\chi^{*} \omega(u, v)= & \left(\chi \circ \gamma^{o}\right)^{*} \omega\left(u_{1}, v_{1}\right) \\
= & (\gamma \circ \chi)^{*} \omega\left(u_{1}, v_{1}\right) \\
= & \gamma^{*} \omega\left(\chi^{\prime} \cdot u_{1}, \chi^{\prime} \cdot v_{1}\right) \\
= & \omega\left(\chi^{\prime} \cdot u_{1}, \chi^{\prime} \cdot v_{1}\right)+F^{*} d \gamma\left(\chi^{\prime} \cdot u_{1}, \chi^{\prime} \cdot v_{1}\right) \\
= & \omega^{o}\left(u_{1}, v_{1}\right)+\pi^{*} d \gamma\left(u_{1}, v_{1}\right) \\
& \left(\chi \text { is symplectic along } 0_{B} \text { and } F \circ \chi=\pi\right) \\
= & \omega^{o}(u, v)
\end{aligned}
$$

Remark 2. The Poincaré lemma relative to a fiber $M_{b}$ shows that $\omega$ is exact (although $H^{2}(M, \mathbb{R})=H^{2}\left(\mathbb{T}^{n}, \mathbb{R}\right)$ is not trivial if $\left.n \geq 2\right) .5$

Let $\lambda$ be a primitive of $\omega$ and $\gamma_{1}(b), \ldots, \gamma_{n}(b)$ be smooth generators of the fundamental group of $M_{b}$ with basepoint $\sigma(b)$, varying smoothly with $b \in B$. Define $I$ by

$$
I_{i}=\oint_{\gamma_{i}(b)} \lambda, \quad i=1, \ldots, n,
$$

and $\theta$ as the (multi-valued) dual variables of $I$ such that $\theta=0$ on $\sigma(B)$.

\footnotetext{
${ }^{5}$ This is assumed in [6] and shown using a first version of the action-angle variables in [16]. If $X$ is Lagrangian, this also follows from the fact that the only symplectic invariant of a neighborhood of $X$ is the diffeomorphism class of $X$ itself [38, 48].
} 
Lemma 5. If $B$ is simply connected, the variables $(I, \theta)$ induce action-angle coordinates $M \rightarrow \mathbb{R}^{n} \times \mathbb{T}^{n}$, which form a diffeomorphism locally in the neighborhood of any fiber of $F$.

Theorem 1 follows from this lemma, by setting $B^{o}=I(M)$.

Proof. If $J=\left(J_{1}, \ldots, J_{n}\right): B \rightarrow \mathbb{R}^{n}$ is a coordinate system over $B$, the natural symplectic coordinates associated with $J$ over $T^{*} B$ is $(J, \varphi)$, with $\varphi_{i}=\partial / \partial J_{i}$, i.e.

$$
\varphi_{i}\left(\beta_{b}\right)=\left.\beta_{b} \cdot \frac{\partial}{\partial J_{i}}\right|_{b}, \quad i=1, \ldots, n, \quad \beta_{b} \in T_{b}^{*} B
$$

We want to compute the coordinates $I_{i}$ in terms of such well chosen coordinates $(J, \varphi)$, in order to see that $(1)$ the $I_{i}$ 's are independent, $(2)(I, \theta)$ is symplectic and (3) $\theta$ is $\mathbb{T}^{n}$-valued.

Since the map $\chi$ is a local diffeomorphism everywhere, the set $L=\chi^{-1}(\sigma(B))$ of elements of $T^{*} B$ acting trivially on $M$ is a submanifold of dimension $n$ (with countably many connected components). Besides, $\chi$ being symplectic and $\sigma(B)$ being Lagrangian, $L$ itself is Lagrangian. Due to lemma 1, the trace of $L$ on a fiber $T_{b}^{*} B$ is a maximal lattice $L_{b}$.

Let $b \in B$. Using $\chi$, lift the loops $\gamma_{i}(b)(i \in\{1, \ldots, n\})$, to paths $\tilde{\gamma}_{i}(b)$ in $T_{b}^{*} B$ starting at $(b, 0)$ and ending at some points $\beta_{i}(b) \in T_{b}^{*} B$. Since $\left(\gamma_{1}(b), \ldots, \gamma_{n}(b)\right)$ is a basis of the fundamental group of $M_{b}$, the so-defined $\left(\beta_{1}(b), \ldots, \beta_{n}(b)\right)$ is a basis of $L_{b}$ over $\mathbb{Z}$. When now $b$ is varied, the covectors $\beta_{i}(b)$ extend to uniquely defined 1 -forms $\beta_{1}, \ldots, \beta_{n}$, whose disjoint images are $n$ connected components of $L$. Since $L$ is Lagrangian, the 1 -forms $\beta_{i}$ are closed: there are functions $J_{1}, \ldots, J_{n}$ over $B$ such that

$$
\beta_{i}=d J_{i}, \quad i=1, \ldots, n .
$$

That the $\beta_{i}$ 's form a basis of the lattice subbundle entails that the $J_{i}$ 's are independent, and thus form a coordinate system over $B$. Define the dual coordinates $\varphi_{1}, \ldots, \varphi_{n}$ (as above for $I$ and $\theta$ ), by letting $\varphi_{i}$ be the tangent vector field $\varphi_{i}=\partial / \partial J_{i}$ over $B$.

Define the primitive

$$
\lambda^{o}=\sum_{1 \leq i \leq n} J_{i} d \varphi_{i}
$$

of the symplectic form $\omega$. Since $\chi$ is symplectic, $\chi^{*} \lambda-\lambda^{o}$ is closed in $T^{*} B$, hence exact: $\chi^{*} \lambda-\lambda^{o}=d S$, for $B$ is simply connected by assumption. Note that $S: M \rightarrow \mathbb{R}$ is $L$-periodic, and, for every $i=1, \ldots, n$, the function

$$
S\left(I, \varphi+\partial / \partial \varphi_{i}\right)-S(I, \varphi)
$$

is constant equal to some $c_{i} \in \mathbb{R}$.

Since for a given $b \in B$ the fiber $T_{b}^{*} B$ is Lagrangian, the integral

$$
I_{1}(b)=\int_{\tilde{\gamma}_{1}(b)} \chi^{*} \lambda
$$


depends on $\tilde{\gamma}_{1}(b)$ only through its homotopy class, and we may replace $\tilde{\gamma}_{1}(b)$ by the path with the same endpoints

$$
\tilde{\gamma}_{1}(b)=\left(J(b),\left(\varphi_{1}, 0, \ldots, 0\right)\right)_{0 \leq \varphi_{1} \leq 1},
$$

and similarly for $I_{2}, \ldots, I_{n}$. Thus, for $i=1, \ldots, n$,

$$
\begin{aligned}
I_{i}(b) & =\int_{\tilde{\gamma}_{i}(b)^{o}} \chi^{*} \lambda \\
& =\int_{\tilde{\gamma}_{i}(b)^{o}}\left(\lambda^{o}+d S\right) \\
& =J_{i}(b)+c_{i} .
\end{aligned}
$$

Consequently, $I_{i}$ differs from $J_{i}$ by a mere constant and $\theta_{i}=\varphi_{i} \circ \chi^{-1}\left(\bmod \mathbb{Z}^{n}\right)$, where $\chi^{-1}$ stand for any inverse branch of $\chi$.

Remark 3 (Adiabatic invariants). The action coordinates $I_{i}$ defined by (1) play an important role in classical dynamics because of their adiabatic invariance, i.e. their invariance under infinitesimally slow perturbations [40]. They also play a crucial role in the Sommerfeld quantization, which is explained by Ehrenfest's "adiabatic hypothesis": quantities which are to be quantized must be adiabatically invariant, because, on perturbing the system, these quantities would have to remain integral multiples of the Planck constant [41].

There are numerous examples illustrating theorem 1, for example in the book [7]. Examples closely related to next section's topic are the Delaunay coordinates (see [13, 44], or [22, Appendix] 6 for a closer viewpoint), or action-angle coordinates of the non-Newtonian Kepler problem [23].

\section{The Poincaré coordinates}

The Poincaré variables are symplectic coordinates in the phase space of the Kepler problem, in the neighborhood of horizontal circular motions. Determining such coordinates departs from the abstract setting of the first section in two respects:

- The Kepler problem is super-integrable in the sense that it has more independent (non commuting) first integrals than degrees of freedom, so the dynamics is degenerate and does not determine in itself a full set of coordinates.

- The action of rotations is degenerate at circular Keplerian ellipses, in the sense that $d H \wedge d C=0$ with the notations below.

\footnotetext{
${ }^{6}$ This appendix is really on the Delaunay coordinates, and does not prove the analyticity of the Poincaré coordinates.
} 
The Poincaré variables are the closer to being action-angle coordinates in this situation. Despite being of prime importance in perturbation theory [2, 14, 21, 43 , (see also more complicated, Deprit-like coordinates of the $N$-body problem in 14 , 42]), they have few complete descriptions in the literature (see [9, 10, 13, 44] for proofs at various levels of precision), all of which are based on Poincaré's computation, through the Delaunay coordinates 7.8 This computation requires a good deal of intuition -which Poincaré did not lack.

Here we aim at providing a slightly more direct construction (although symplecticity is always more simple to check by relating the Poincaré coordinates to the Delaunay coordinates), trying to find out the definition of the coordinates at the same time as proving their properties.

In the sequel, we will set $\mathbb{T}^{n}=\mathbb{R}^{n} / 2 \pi \mathbb{Z}^{n}$ (as opposed to $\mathbb{R}^{n} / \mathbb{Z}^{n}$ as in the first section) for the sake of convenience.

\subsection{Reminder on the plane Kepler problem}

We start with the plane problem because it is an interesting intermediate step, with simpler computations. In this section, we recall some elementary (non symplectic) facts.

Consider the equation

$$
\ddot{q}=-\frac{q}{r^{3}}, \quad q \in \mathbb{C} \backslash\{0\}, \quad r=|q| ;
$$

references are so numerous that we give up advising any one of them. The phase space is the set $\{(\dot{q}, q)\}=\mathbb{C} \times(\mathbb{C} \backslash\{0\})$.

- The angular momentum

$$
C=\operatorname{Im}(\bar{q} \dot{q})=x \dot{y}-y \dot{x}
$$

is preserved, as in any central force problem (Kepler's second law).

- The eccentricity vector

$$
E=\frac{q}{r}+i C \dot{q}
$$

too is preserved, this time in contrast to other central forces than Newton's. The equation of an orbit can be obtained by eliminating the velocity from $C$ and $E$ :

$$
\operatorname{Re}(\bar{E} q)=r-C^{2}
$$

which is the equation of the conic with a focus at the origin (Kepler's first law), of eccentricity $\epsilon=|E|$ and with directrix $D: \operatorname{Re}(\bar{E} q)+C^{2}=0$.

\footnotetext{
${ }^{7}$ In the first edition of [1], the Delaunay and Poincaré coordinates are wrongly found non symplectic!

${ }^{8}$ In the unpublished note [20] on the plane Poincaré coordinates, it is wrongly claimed that the analyticity of the Poincaré coordinates was not proved by Poincaré. Poincaré did prove that the inverse map is analytic (and in particular, that the Hamiltonian is an analytic function of the Poincaré coordinates), which, due to the inverse function theorem, is equivalent to proving that the coordinate map itself is analytic.
} 
- The Hamiltonian

$$
H=\frac{|\dot{q}|^{2}}{2}-\frac{1}{r}
$$

(denoted $H$ by Lagrange in reference to Huygens [28]) too is a first integral. The dynamics is that of the Hamiltonian vector field of $H$ with respect to the standard symplectic form, $\omega=\operatorname{Re}(d \bar{p} \wedge d q)$.

Because of the symmetry about the origin, it is useful to switch to polar coordinates. The cotangent map of the "polar coordinate" map $(r, \theta) \mapsto q=r e^{i \theta}$ is the diffeomorphism 9

$\mathrm{Pol}: \mathbb{R}^{2} \times \mathbb{R}_{*}^{+} \times \mathbb{T} \rightarrow \mathbb{C} \times(\mathbb{C} \backslash\{0\}), \quad(R, \Theta, r, \theta) \mapsto(\dot{q}, q)=\left(\left(R+i \frac{\Theta}{r}\right) e^{i \theta}, r e^{i \theta}\right)$.

The (pull-backs by Pol of the) first integrals are

$$
H=\frac{R^{2}}{2}+\frac{\Theta^{2}}{2 r^{2}}-\frac{1}{r}, \quad C=\Theta \quad \text { and } \quad E=e^{i \theta}\left(1-\frac{\Theta^{2}}{r}+i \Theta R\right) .
$$

The polar representation of $E$ is

$$
E=\sqrt{1+2 H \Theta^{2}} e^{i g},
$$

where the argument $g$ is easily computed when $R=0$ : it is the argument of the pericenter of the conic (when the conic is a circle and the pericenter thus not defined, $E=0$ is well defined, of course).

From now on, we restrict to negative energies. If $a$ is the semi major axis, $r$ varies between $a(1-\epsilon)$ and $a(1+\epsilon)$. These two extremal values are the roots of the quadratic equation $H=\frac{C^{2}}{2 r^{2}}-\frac{1}{r}$, so their sum and product are

$$
2 a=-\frac{1}{2 H} \quad \text { and } \quad a^{2}\left(1-\epsilon^{2}\right)=-\frac{C^{2}}{2 H} \text {. }
$$

Hence, using (5),

$$
H=-\frac{1}{2 a}, \quad C=\sqrt{a\left(1-\epsilon^{2}\right)}, \quad \text { and } \quad E=\epsilon e^{i g} ;
$$

we will use that these functions are analytic.

Now, according to Kepler's second law, the area swept by $q$ grows with constant speed $C / 2$. Since the area of the ellipse is $\pi a b=\pi a^{2} \sqrt{1-\epsilon^{2}}$, the following relation holds between the period $T$ and the elliptic elements:

$$
\frac{\pi a^{2} \sqrt{1-\epsilon^{2}}}{T}=\frac{C}{2}
$$

and, thanks to the remarkable disappearance of the eccentricity (use (6) ), so does Kepler's third law:

$$
T=2 \pi a^{3 / 2}
$$

We will also use three classical angles, defined when the ellipse is not circular 10

\footnotetext{
${ }^{9}$ Another way to compute the conjugate variable of $\theta$ is to think of $\Theta$ as the momentum $i(X) p d q$ of the rotational vector field $X$.

${ }^{10}$ Notations here follow Poincaré's quite closely.
} 


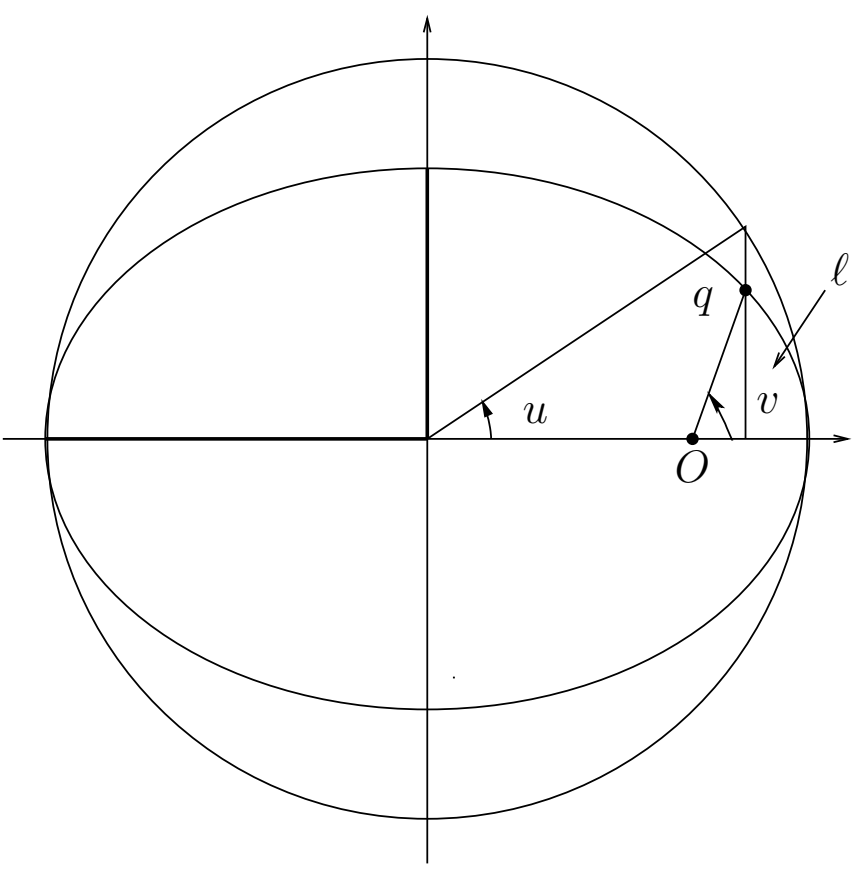

Figure 1: Eccentric, mean and true anomalies $(u, v, \ell)$

- the mean anomaly $\ell$ is the angle which is proportional to the area, counting from the pericenter (Kepler's second law says that $\ell$ increases linearly with time)

- the eccentric anomaly $u$ is defined on figure 1

- the true anomaly $v=\theta-g$.

Elementary geometry yields Kepler's fundamental equation

$$
\ell=u-\epsilon \sin u,
$$

which shows that $q$ is a transcendant function of $\ell$ (see Newton's proof in [5]), that $u$ does vary around the whole circle, etc.

We will need one more fact. The anomalies $u, v$ or $\ell$ are not analytic, nor even defined, at circular motions. In contrast, the eccentric longitude $w=u+g$ is analytic. Indeed, elementary geometry again shows that

$$
\left\{\begin{array}{l}
r \cos v=a(\cos u-\epsilon) \\
r \sin v=a \sqrt{1-\epsilon^{2}} \sin u .
\end{array}\right.
$$

Solving these two equations for $\cos u$ and $\sin u$ allows us to express trigonometric functions of $w$ in terms of $v=\theta-g$ : for example,

$$
\begin{aligned}
\cos w & =\left(\frac{r}{a} \cos (\theta-g)+\epsilon\right) \cos g-\frac{r}{a \sqrt{1-\epsilon^{2}}} \sin (\theta-g) \sin g \\
& =\frac{r}{\sqrt{a} \Theta}\left[\cos \theta+\left(\frac{\Theta}{\sqrt{a}}-1\right)\left(\cos ^{2} g \cos \theta+\cos g \sin g \sin \theta\right)\right]+\epsilon \cos g ;
\end{aligned}
$$


the right hand side is real analytic because $\frac{\Theta}{\sqrt{a}}-1=\sqrt{1-\epsilon^{2}}-1=O\left(\epsilon^{2}\right)$, and $E=\epsilon e^{i g}$ is analytic. The function $\sin w$ can be seen to be real analytic similarly. So, $w$ is real analytic.

\subsection{Plane Poincaré variables}

Consider the plane Kepler problem with negative energy and, say, positive angular momentum. The phase space is diffeomorphic to $\mathbb{R}^{3} \times \mathbb{T}^{1}$ and has coordinates $(R, r, \Theta, \theta)$ and symplectic form $\omega=d R \wedge d r+d \Theta \wedge d \theta$.

Keplerian action variable $\Lambda$ First consider the problem reduced by rotations, in the symplectic space $\mathcal{K}=\{(R, r)\} \simeq \mathbb{R}^{2}$, with the angular momentum $\Theta$ as a parameter. The reduced Hamiltonian $H=H_{\Theta}(R, r)$ has an elliptic singularity at $(R, r)=\left(0, \Theta^{2}\right)$, corresponding to circular motions. Locally outside the singularity, the energy $H$ and the time $t$ (counted from some section of the flow of $H$, which we do not want to specify at this stage) form some symplectic coordinates. We would like to switch to some coordinates $(\Lambda, \lambda)$ where the action is a well chosen function of $H$, so that the dual coordinate $\lambda$ be an angle, defined modulo $2 \pi$ :

$$
d H \wedge d t=d \Lambda \wedge d \lambda
$$

where $\Lambda=\Lambda(H)$ and $\lambda=\frac{2 \pi}{T} t$. Hence,

$$
d H \wedge d t=\frac{2 \pi}{T} \Lambda^{\prime}(H) d H \wedge d t
$$

or

$$
\Lambda^{\prime}=\frac{T}{2 \pi}=a^{3 / 2}=\left(-\frac{1}{2 H}\right)^{3 / 2},
$$

or, if we choose the primitive vanishing at $H=-\infty$,

$$
\Lambda=\frac{1}{\sqrt{2}}(-H)^{-1 / 2}=\sqrt{a} .
$$

Up to now, $\lambda$, is only determined up to the addition of a first integral.

Remark 4. The above computation of $\Lambda^{\prime}$ can be recovered from the expression of the action coordinate given in the previous section (up to a factor $2 \pi$ due to the fact that we then took circles of length 1 ). Indeed, denoting by $X_{t}$ the (globally defined) Hamiltonian vector field of $t$, by $\left(\phi_{H}\right)$ its flow (with "time" $H$ ), and by $H_{0}=\frac{1}{2 \Theta^{2}}$ the value of the Hamiltonian at circular motions,

$$
\begin{aligned}
\Lambda^{\prime}(H) & =\frac{1}{2 \pi} X_{t} \cdot \oint_{H=H_{0}} \phi_{H-H_{0}}^{*} R d r \\
& \left.=\frac{1}{2 \pi} \oint_{H=H_{0}} \phi_{H-H_{0}}^{*} \mathcal{L}_{X_{t}} R d r\right) \\
& =\frac{1}{2 \pi} \oint_{H=H_{0}} \phi_{H-H_{0}}^{*}\left(d t+d i_{X_{t}} R d r\right) \quad \text { (Cartan formula) } \\
& =\frac{T}{2 \pi}
\end{aligned}
$$


The Keplerian action variable $\Lambda$ lifts to an analytic variable in the non-reduced phase space (analyticity follows from (6) ).

Eccentric variable $F$ One could check that the symplectic transformation $(R, r) \mapsto(\Lambda, \lambda)$ in the reduced space lifts to an essentially unique symplectic transformation $(R, r, \Theta, \theta) \mapsto\left(\Lambda, \lambda, F_{1}, F_{2}\right)$ in the full phase space, possibly using a generating function. But this computation is more involved than necessary (a similar computation is made in [23] in the non-Newtonian two-body problem at the second order in the eccentricity). We will make a much shorter computation, completed by geometric arguments.

Consider the space $\mathcal{E}$ of Keplerian ellipses of fixed energy $H<0$. For an ellipse in the plane, to be Keplerian means that it is oriented and has a focus at the origin. In addition to that, Keplerian ellipses in $\mathcal{E}$ have a fixed semi major axis. Including degenerate ellipses corresponding to collision orbits of eccentricity $1, \mathcal{E}$ is diffeomorphic to $S^{2}$. Outside the poles $\{N, S\}$ corresponding to circular ellipses, it bears the coordinates $(\Theta, g)$, where $g$ is the argument of the pericenter. Since the flow of $\Theta=C$ consists of rigid rotations in the plane, the Poisson bracket of $\Theta$ and $g$ is $\{\Theta, g\}=1$, hence the symplectic form induced from $\omega$ on $\mathcal{E} \backslash\{N, S\}$ is $\omega_{\mathcal{E}}=d \Theta \wedge d g$.

We will focus on the open hemisphere $\mathcal{E}^{+}$of $\mathcal{E}$ consisting of direct, non-degenerate ellipses; this domain is defined in $\mathcal{E}$ by the inequality $\Theta>011$ Over $\mathcal{E}^{+}$, the excentricity vector $E$ is a (complex-valued) real analytic coordinate, unfortunately not symplectic, since, using the expression (5), we get

$$
\frac{1}{2 i} d \bar{E} \wedge d E=-\frac{\Theta}{\Lambda^{2}} d \Theta \wedge d g
$$

Let us first look to the case $\Lambda=1$. We will look for a real analytic symplectic coordinate $F$ obtained by multiplying $\bar{E}$ (not $E$ because of the negative sign in (8)) by a positive real analytic function $f$ of $\Theta \in] 0,1]$ :

$$
F=f(\Theta) \bar{E} .
$$

Direct computation yields

$$
\frac{1}{2 i} d \bar{F} \wedge d F=\left[\Theta f(\Theta)^{2}-\left(1-\Theta^{2}\right) f(\Theta) f^{\prime}(\Theta)\right] d \Theta \wedge d g .
$$

The requirement that $F$ be symplectic is equivalent to imposing that the expression in brackets equal 1 . In the unknown $\varphi=f^{2}$, the equation becomes

$$
\left(1-\Theta^{2}\right) \varphi^{\prime}(\Theta)=2(\Theta \varphi(\Theta)-1)
$$

Solutions are of the form

$$
\varphi(\Theta)=\frac{2 \Theta+c}{1-\Theta^{2}}, \quad c \in \mathbb{R}
$$

\footnotetext{
${ }^{11}$ One can similarly define dual Poincaré coordinates over the hemisphere of negatively oriented ellipses.
} 
The only solution analytic (and even continuous) at $\Theta=1$ corresponds to $c=-2$ i.e., $\varphi(\Theta)=\frac{2}{1+\Theta}$ i.e., $F=\sqrt{2(1-\Theta)} e^{-i g}$. For a general value of the parameter $\Lambda$, by homogeneity ( $F$ having the dimension of $\sqrt{\Lambda}$ and $\Theta$ having that of $\Lambda$ ), one gets

$$
F=\sqrt{2 \Lambda} \sqrt{1-\frac{\Theta}{\Lambda}} e^{-i g}=\sqrt{2(\Lambda-\Theta)} e^{-i g}
$$

Lemma 6. The coordinate $F$ is real-analytic and symplectic over $\mathcal{E}^{+}$.

That $F$ be symplectic has already been proved. That it is analytic follows from the formula

$$
F=\sqrt{\frac{2 \Lambda}{1+\frac{\Theta}{\Lambda}}} \bar{E} .
$$

Mean longitude $\lambda$ The variable $F$ can be lifted to the full phase space of the plane Kepler problem. We need to show that the coordinates $(\Lambda, \lambda, F)$ are analytic and symplectic, provided that we make some adequate choice of a real analytic section of the flow of $\Lambda$ to define $\lambda=\ell+c s t$; this choice of constant corresponds to choosing an analytic Lagrangian section in lemma 3. Define $\lambda$ as the mean longitude

$$
\lambda=\ell+g
$$

this choice is primarily motivated by the first argument given in the proof of the following statement.

Lemma 7. The coordinate system $(\Lambda, \lambda, F)$ is real analytic and symplectic in the neighborhood of direct circular Keplerian motions.

Only $\lambda$ is not yet known to be analytic. Adding $g$ to Kepler'equation (7) yields

$$
\lambda=w-\epsilon \sin (w-g)=w+\operatorname{Im}\left(E e^{-i w}\right),
$$

where both $E$ and $w$ are analytic. So $\lambda$ is analytic.

We need to show that the coordinate system is symplectic. By continuity, it is enough to check this outside circular motions. Recall that the "symplectic polar map"

$$
\mathbb{R}_{*}^{+} \times \mathbb{T}^{1} \rightarrow \mathbb{C}, \quad(\rho, \phi) \mapsto z=\sqrt{2 \rho} e^{i \phi}
$$

is symplectic: $\frac{1}{2 i} F^{*}(d \bar{z} \wedge d z)=d \rho \wedge d \phi$. So, the question reduces to checking that $(\Lambda, \lambda, \Lambda-\Theta,-g)$ is symplectic, or, equivalently, that the Delaunay coordinates $(\Lambda, \ell, \Theta, g)$ are symplectic.

Since the matrix of the symplectic form is the inverse of the matrix of the Poisson structure, we will check that the Poisson brackets are given by the standard matrix. We know that $\{\Lambda, \Theta\}=\{\Lambda, \ell\}=\{\Theta, g\}=1$. Three Poisson brackets remain to be checked:

- $\{\Theta, \ell\}=0$ because the flow of $\Theta$ acts by diagonal rotations in the phase space. 
- $\{\Lambda, g\}=0$ because $g$ is a first integral of $H=H(\Lambda)$.

- $\{\ell, g\}$ does require some computation: according to Jacobi's identity,

$$
\{\Lambda,\{\ell, g\}\}=\{\Theta,\{\ell, g\}\}=0
$$

so it is enough to prove that $\{\ell, g\}=0$ on the codimension-2 submanifold $\ell=g=0(\bmod 2 \pi)$, a section of the $L$ - and $\Theta$-flows. So, without loss of generality we may thus assume that the body is on the major axis and that the major axis itself is the first coordinate axis. Then the partial derivatives of $\ell$ and $g$ with respect of $x$ or $p_{y}$ are zero, and

$$
\{\ell, g\}=(\underbrace{\frac{\partial \ell}{\partial x}}_{=0} \frac{\partial g}{\partial p_{x}}-\frac{\partial \ell}{\partial p_{x}} \underbrace{\frac{\partial g}{\partial x}}_{=0})+(\frac{\partial \ell}{\partial y} \underbrace{\frac{\partial g}{\partial p_{y}}}_{=0}-\underbrace{\frac{\partial \ell}{\partial p_{y}}}_{=0} \frac{\partial g}{\partial y})=0 .
$$

\subsection{Reminder on the spatial Kepler problem}

Now consider the same equation as in (2) but with $q=(x, y, z) \in \mathbb{R}^{3} \backslash\{0\}$. We will again restrict to negative energy

$$
H=\frac{\|\dot{q}\|}{2}-\frac{1}{r}
$$

and non collision motions $(q \wedge \dot{q} \neq 0)$. Due to the equivariance of the equation by orthogonal symmetries, a solution $q(t)$ is drawn on the vector plane generated by $q(0)$ and $\dot{q}(0)$.

Redefine the angular momentum and the eccentricity vector respectively as

$$
C=q \wedge \dot{q} \in \mathbb{R}^{3} \wedge \mathbb{R}^{3} \equiv \mathbb{R}^{3} \quad \text { and } \quad \tilde{E}=\frac{q}{r}+i(\dot{q}) C,
$$

with $r=\|q\|$ and $i(\dot{q}) C=(\dot{q} \cdot q) \dot{q}-\dot{q}^{2} q$.

We will need extensions of $\epsilon e^{i g}$ and of the eccentric longitude $w$ in space. Let $\mathfrak{R}_{q}(\alpha) \in S O_{3}$ be the rotation around a vector $q \in \mathbb{R}^{3}$ of angle $\alpha, \mathfrak{R}_{x}(\alpha)=$ $\mathfrak{R}_{(1,0,0)}(\alpha)$, and similarly for rotations around the two other vectors of the canonical basis of $\mathbb{R}^{3}$. When the orbital plane is not the "horizontal" plane ( $x y$-plane), define

- the inclination $\iota$, or the angle of the orbital plane with respect to the horizontal plane,

- $\Delta$, the oriented direction of the ascending node (half line from the center of attraction to the point of the Keplerian ellipse where $z=0$ and $\dot{z}>0$ ),

- the longitude of the node $\varphi$, or the angle between the $x$-axis and $\Delta$. 
Since $\tilde{E}$ lies in the orbital plane, $\mathfrak{R}_{\Delta}(-\iota) \tilde{E}$ is horizontal. The fortunate fact is that this rotation matrix is an analytic function of $q$ and $\dot{q}$. Indeed, using a classical decomposition (see [31]), we see that

$$
\begin{aligned}
\mathfrak{R}_{\Delta}(\iota) & =\mathfrak{R}(\varphi) \mathfrak{R}(\iota / 2) \mathfrak{R}(\iota / 2) \mathfrak{R}(-\varphi) \\
& =\left(\begin{array}{ccc}
1-2 \alpha^{2} & 2 \alpha \beta & -2 \alpha \gamma \\
2 \alpha \beta & 1-2 \beta^{2} & 2 \beta \gamma \\
2 \alpha \gamma & -2 \beta \gamma & 1-2 \alpha^{2}-2 \beta^{2}
\end{array}\right),
\end{aligned}
$$

where we have used the auxiliary notation

$$
\alpha=\frac{C_{x}}{2 \gamma\|C\|}, \quad \beta=-\frac{C_{y}}{2 \gamma \Theta}, \quad \gamma=\cos \frac{\iota}{2}=\sqrt{\frac{1+C_{z} / \Theta}{2}}, \quad \Theta=\|C\| .
$$

Identifying $\mathfrak{R}_{\Delta}(-\iota) \tilde{E} \in \mathbb{R}^{2} \times\{0\}$ to a complex number, define the analytic variable

$$
E=\mathfrak{R}_{\Delta}(-\iota) \tilde{E}=\epsilon e^{i(g+\varphi)} \in \mathbb{C} .
$$

Moreover, the computation analogous to the one made in the plane shows that now the eccentric longitude

$$
w=u+g+\varphi
$$

is analytic.

\subsection{Spatial Poincaré coordinates}

Horizontal variables The plane coordinates $(\Lambda, \lambda, F)$ extend to real analytic variables in a neighborhood of circular coplanar Keplerian motions in the spatial phase space $\{(\dot{q}, q)\}=\mathbb{R}^{3} \times\left(\mathbb{R}^{3} \backslash\{0\}\right)$, in the following manner:

- $\Lambda=\sqrt{a}$, where $a$ is the semi major axis. Due to the invariance of the Hamiltonian by rotations, the relation $H=-\frac{1}{2 a}$ still holds in space, showing that $a$ and thus $\Lambda$ are real analytic.

- $\lambda=\ell+g+\varphi$.

- $F=\sqrt{2(\Lambda-\Theta)} e^{-i(g+\varphi)}=\sqrt{\frac{2 \Lambda}{1+\frac{\Theta}{\Lambda}}} \bar{E}$

Only $\lambda$ is not obviously analytic. But, this follows from Kepler's equation 7 , to which one can add $g+\varphi$ (instead of $g$ in the plane), to get

$$
\lambda=w-\epsilon \sin (w-\varphi-g)=w+\operatorname{Im}\left(E e^{-i w}\right),
$$

and from the fact that $E$ and the eccentric longitude $w$ are analytic. 
Oblique variable The complex-valued variable

$$
\Gamma=C_{x}+i C_{y}=-i \sqrt{\Theta^{2}-\Phi^{2}} e^{i \varphi}, \quad \text { with } \quad \Phi=C_{z}=\Theta \cos \iota,
$$

can be added to $(\Lambda, \lambda, F)$ to form an analytic coordinate system in space. Unfortunately, $(\Lambda, \lambda, F, \Gamma)$ is not a symplectic since, in restriction to the tangent space generated by $\partial / \partial z$ and $\partial / \partial \dot{z}$,

$$
\frac{1}{2 i} d \bar{\Gamma} \wedge d \Gamma=C_{z} d \dot{z} \wedge d z
$$

Looking for a symplectic modification of $\Gamma$ of the form $G=f(\Theta, \Phi) \bar{\Gamma}$ by carrying out an analogous computation of the symplectic form as after (9), using spherical coordinates, one can find

$$
G=\sqrt{2(\Theta-\Phi)} e^{-i \varphi}
$$

another way to build spatial coordinates from the plane ones is described in [50]. $G$ is analytic because

$$
G=\frac{\sqrt{2}}{i} \frac{\bar{\Gamma}}{\sqrt{\Theta+\Phi}}
$$

Theorem 2. The coordinate system $(\Lambda, \lambda, F, G)$ with

$$
\left\{\begin{array}{l}
\Lambda=\sqrt{a} \\
\lambda=\ell+g+\varphi \\
F=\sqrt{2(\Lambda-\Theta)} e^{-i(g+\varphi)} \\
G=\sqrt{2(\Theta-\Phi)} e^{-i \varphi}
\end{array}\right.
$$

is analytic and symplectic in the neighborhood of direct circular horizontal Keplerian motions.

Only the property of being sympletic remains to be proved. Since the map (12) is symplectic, by continuity it suffices to show that the coordinate system

$$
(\Lambda, \lambda, \Lambda-\Theta,-g-\varphi, \Theta-\Phi,-\varphi)
$$

(defined outside horizontal or circular motions) is symplectic, or, equivalently, that the Delaunay coordinates

$$
(\Lambda, \ell, \Theta, g, \Phi, \varphi)
$$

are symplectic.

- Poisson brackets with $\Lambda, \Theta$ and $\Phi$ are all 0 , except $\{\Lambda, \ell\}=\{\Theta, g\}=$ $\{\Phi, \varphi\}=1$ (we know the flows of $\Lambda, \Theta$ and $\Phi$ ).

- The three Poisson brackets between pairs of angles among $\ell, g$ and $\varphi$ vanish. Indeed, as in the plane, the Jacobi identity shows that it is enough to check those Poisson brackets on the submanifold $\{\ell=g=\varphi=0(\bmod \pi)\}$. But on this submanifold the partial derivatives of any of the angles with respect to $x, p_{y}$ or $p_{z}$ vanish. 
This completes the proof of theorem 2 .

Thank you to A. Albouy and A. Chenciner for their critical reviewing and to $P$. Robutel for advertising the eccentric longitude to me. The author has been partially supported by the French ANR (projet ANR-10-BLAN 0102 DynPDE).

\section{References}

[1] R. Abraham and J. E. Marsden. Foundations of mechanics. Benjamin/Cummings Publishing Co. Inc. Advanced Book Program, Reading, Mass., 1978. Second edition, revised and enlarged, With the assistance of T. Raţiu and R. Cushman.

[2] V. I. Arnold. Small denominators and problems of stability of motion in classical and celestial mechanics. Uspehi Mat. Nauk, $18(6$ (114)):91-192, 1963.

[3] V. I. Arnold. A theorem of Liouville concerning integrable problems of dynamics. Sibirsk. Mat. Ž., 4:471-474, 1963.

[4] V. I. Arnold. Mathematical methods of classical mechanics, volume 60 of Graduate Texts in Mathematics. Springer-Verlag, New York, 1989. Translated from the 1974 Russian original by K. Vogtmann and A. Weinstein, Corrected reprint of the second (1989) edition.

[5] V. I. Arnold. Kepler's second law and the topology of Abelian integrals (according to Newton) [Kvant 1987, no. 12, 17-21]. In Kvant selecta: algebra and analysis, II, volume 15 of Math. World, pages 131-140. Amer. Math. Soc., Providence, RI, 1999.

[6] V. I. Arnold and A. Avez. Problèmes ergodiques de la mécanique classique. Monographies Internationales de Mathématiques Modernes, No. 9. GauthierVillars, Éditeur, Paris, 1967.

[7] V. I. Arnold, V. V. Kozlov, and A. I. Neishtadt. Mathematical aspects of classical and celestial mechanics, volume 3 of Encyclopaedia of Mathematical Sciences. Springer-Verlag, Berlin, third edition, 2006. Translated from the Russian original by E. Khukhro.

[8] M. Artin. Algebra. Prentice Hall Inc., Englewood Cliffs, NJ, 1991.

[9] L. Biasco, L. Chierchia, and E. Valdinoci. Elliptic two-dimensional invariant tori for the planetary three-body problem. Arch. Ration. Mech. Anal., 170(2):91-135, 2003.

[10] L. Biasco, L. Chierchia, and E. Valdinoci. Corrigendum to: "Elliptic twodimensional invariant tori for the planetary three-body problem" [Arch. Ration. Mech. Anal. 170 (2003), no. 2, 91-135; mr2017886]. Arch. Ration. Mech. Anal., 180(3):507-509, 2006. 
[11] J. M. Burgers. Die adiabatischen Invarianten bedingt periodischer Systeme. Annalen der Physik, 357(2):195-202, 1917.

[12] L. T. Butler and A. Sorrentino. Weak Liouville-Arnold theorems and their implications. Comm. Math. Phys., 315(1):109-133, 2012.

[13] A. Chenciner. Intégration du problème de Kepler par la méthode de HamiltonJacobi. Technical report, Bureau des Longitudes, 1989.

[14] L. Chierchia and G. Pinzari. The planetary $N$-body problem: symplectic foliation, reductions and invariant tori. Invent. Math., 186(1):1-77, 2011.

[15] R. H. Cushman and L. M. Bates. Global aspects of classical integrable systems. Birkhäuser Verlag, Basel, 1997.

[16] J. J. Duistermaat. On global action-angle coordinates. Comm. Pure Appl. Math., 33(6):687-706, 1980.

[17] C. Ehresmann. Les connexions infinitésimales dans un espace fibré différentiable. In Séminaire Bourbaki, Vol. 1, pages Exp. No. 24 (1950), 153-168. Soc. Math. France, Paris, 1995.

[18] A. Einstein. Zum quantensatz von Sommerfeld und Epstein. Deutsche Physikalische Gesellschaft, 19:82-92, 1917.

[19] P. Epstein. Hamilton-Jacobische Funktion und Quantentheorie. Verhandlungen der Deutschen Physicalischen Gesellschaft, 19:116-129, 1917.

[20] Francesco Fasso and Tudor Ratiu. Proof of the analyticity of the poincaré elements, 2000.

[21] J. Féjoz. Démonstration du 'théorème d'Arnold' sur la stabilité du système planétaire (d'après Herman). Ergodic Theory Dynam. Systems, 24(5):15211582, 2004.

[22] J. Féjoz. On "Arnold's theorem" in celestial mechanics -a summary with an appendix on the Poincaré coordinates. Discrete and Continuous Dynamical Systems, 33:3555-3565, 2013.

[23] J. Féjoz and L. Kaczmarek. Sur le théorème de Bertrand (d'après Michael Herman). Ergodic Theory Dynam. Systems, 24(5):1583-1589, 2004.

[24] V. Guillemin and S. Sternberg. Symplectic techniques in physics. Cambridge University Press, Cambridge, second edition, 1990.

[25] C.G.J. Jacobi, C.W. Borchardt, and A. Clebsch. Vorlesungen über Dynamik. G. Reimer, 1866.

[26] R. Jost. Winkel und Wirkungsvariable für allgemeine mecanische systeme. Helvetica Physica Acta, 41:965-968, 1968. 
[27] V. V. Kozlov and N. N. Kolesnikov. Integrability of Hamiltonian systems. Vestnik Moskov. Univ. Ser. I Mat. Mekh., 6:88-91, 109, 1979.

[28] J.-L. Lagrange. Mécanique analytique. Gauthier-Villars, 1888. Quatrième édition en deux volumes. Euvres complètes (Gauthier-Villars), volumes XI et XII. (Première édition en 1808).

[29] L. D. Landau and E. M. Lifshitz. Mechanics. Course of Theoretical Physics, Vol. 1. Translated from the Russian by J. B. Bell. Pergamon Press, Oxford, 1960 .

[30] P. Langevin and M. de Broglie, editors. La théorie du rayonnement et les quanta, chapter Discussion du rapport de M. Planck, pages 116-117. Gauthiers-Villars (Paris), 1911.

[31] J. Laskar. Variables elliptiques. Notes de cours de l'Observatoire de Paris, 2004.

[32] D. Leborgne. Calcul différentiel et géométrie. Presses Universitaires de France, Paris, 1982.

[33] E. Lerman, R. Montgomery, and R. Sjamaar. Examples of singular reduction. In Symplectic geometry, volume 192 of London Math. Soc. Lecture Note Ser., pages 127-155. Cambridge Univ. Press, Cambridge, 1993.

[34] T. Levi-Civita. Sugli invariantici adiabatici. Tratto dal Resoconto del Congresso Nazionale dei Fsici, 1927.

[35] P. Libermann and C.-M. Marle. Géométrie symplectique, bases théoriques de la mécanique. Tome IV, volume 21 of Publications Mathématiques de l'Université Paris VII. Université de Paris VII, Paris, 1987.

[36] J. Liouville. Note sur l'intégration des équations différentielles de la Dynamique. J. mathématiques pures et appliquées, I(20):137-138, 1855. Note présentée au Bureau des Longitudes le 29 juin 1953.

[37] L. Markus and K. R. Meyer. Generic Hamiltonian dynamical systems are neither integrable nor ergodic. American Mathematical Society, Providence, R.I., 1974. Memoirs of the American Mathematical Society, No. 144.

[38] D. McDuff and D. Salamon. Introduction to symplectic topology. Oxford Mathematical Monographs. The Clarendon Press Oxford University Press, New York, second edition, 1998.

[39] H. Mineur. Sur les systèmes mécaniques dans lesquels figurent des paramètres fonctions du temps. J. École polytechnique, pages 173-191, 237-270, 1937.

[40] A. Neishtadt. Averaging method and adiabatic invariants. In Hamiltonian dynamical systems and applications, NATO Sci. Peace Secur. Ser. B Phys. Biophys., pages 53-66. Springer, Dordrecht, 2008. 
[41] W. Pauli. Wave Mechanics. Number vol. 5 in Pauli Lectures on Physics. Dover, 2000.

[42] G. Pinzari. On the Kolmogorov Set for Many-Body Problems. PhD thesis, Universitá di Roma Tre, 2009.

[43] H. Poincaré. Les méthodes nouvelles de la mécanique céleste. Gauthier-Villars, 1892.

[44] H. Poincaré. Leçons de mécanique céleste. Gauthier-Villars, 1905.

[45] S. Sternberg. Lectures on Differential Geometry. AMS Chelsea Publishing Series. American Mathematical Society, 1983.

[46] S. Sternberg. Group theory and physics. Cambridge University Press, Cambridge, 1994.

[47] R.H. Weber, R. Gans, F.A. Schulze, and P. Hertz. Repertorium der Physik. B.G. Teubner, 1916.

[48] A. Weinstein. Symplectic manifolds and their Lagrangian submanifolds. Advances in Math., 6:329-346 (1971), 1971.

[49] N. M. J. Woodhouse. Geometric quantization. Oxford Mathematical Monographs. The Clarendon Press, Oxford University Press, New York, second edition, 1992.

[50] L. Zhao. Quasi-periodic motions in the spatial three-body problem. PhD thesis, Université Paris-Diderot, 2013. 